\title{
Finanzpolitische Exit-Strategien und die europäische Schuldenkrise
}

Fast alle internationalen Organisationen wie EU-Kommission, OECD und IWF plädieren angesichts der Eurokrise für einen möglichst schnellen finanzpolitischen „Exit“, also das schnelle Umschwenken auf einen restriktiven finanzpolitischen Kurs. Ist ein solcher Kurs tatsächlich Erfolg versprechend? Welche Risiken für Wachstum und Beschäftigung birgt er? Wie könnten eine alternative Exit-Strategie und eine wirtschafts- und finanzpolitische Konzeption aussehen, die Wachstum, Beschäftigung und Sozialstaat stärken?

\section{Einleitung}

Die Banken- und Finanzkrise der Jahre 2007 bis 2009 hatte viele Gründe: unkontrollierte Liberalisierung und Globalisierung, riskante Innovationen, Blindheit und Gier der Finanzmärkte und -institutionen, explodierende, nach Rendite und Liquidität suchende Kapitalmengen und falsche makroökonomische Strategien in „neomerkantilistischen “ und angelsächsischen Ländern. Geldpolitische Maßnahmen hatten private Schulden wie Kreditund Immobilienblasen aufgebläht und ein Wachstum ohne Lohnzuwachs und sozialen Ausgleich entstehen lassen (Mathieu/ Sterdyniak 2010).

Die öffentlichen Schuldenstände und -defizite sind dagegen nicht für die Krise verantwortlich. 2007 beliefen sich die staatlichen Defizite auf durchschnittlich gerade einmal 1,3\% des Bruttoinlandsprodukts (BIP) in den OECD-Ländern, im Euroraum sogar nur auf 0,6 \% des BIP. Der rasante Anstieg der Staatsschulden und -defizite entstand durch die Rettung des Finanzsystems, den anschließenden Einbruch bei den Steuereinnahmen und die Notwendigkeit von Konjunkturprogrammen zur Stützung der Wirtschaft. Er kam also nicht durch staatliche Verschwendung zustande. Vielmehr hat die Finanzkrise plastisch vor Augen geführt, dass eine aktive Finanzpolitik, staatliche Eingriffe und Regulierungen unabdingbar sind.

Seit 2010 nähren die Finanzmärkte Zweifel an der Tragfähigkeit der öffentlichen Finanzen und verlangen ein starkes Zurückfahren der Haushaltsdefizite, obwohl diese zur Konjunkturstützung notwendig sind. Im Euroraum ist dieses Thema besonders akut, da das Rahmenwerk des Maastrichtvertrages gescheitert ist. Seit Ende 2008 wird auf den Finanzmärkten über das Auseinanderbrechen des Euroraums spekuliert. Sollen die Euroländer ihre Staatsdefizite zurückfahren, auch auf die Gefahr hin, die wirtschaftliche Erholung auszubremsen? Wird die Finanzkrise es den herrschenden Eliten und EU-Technokraten gestatten, restriktive Wirtschaftspolitik, liberale Reformen und Kürzungen von Sozialausgaben durchzusetzen? Oder können die EU-Institutionen oder die Mitgliedstaaten bessere wirtschaftspolitische Rahmenbedingungen einführen?

\section{Finanzpolitik vor der Krise}

Noch 2007, vor dem Ausbruch der Finanzkrise, lagen die Staatsfinanzen der meisten Euroländer mit ihren Primärhaushaltssalden im Plus: 2 \% des BIP im Euroraum (Tabelle 1). Nur Frankreich lag leicht unterhalb des zur Stabilisierung der Schuldenstandsquote notwendigen Saldos. Länder wie Spanien, Griechenland und Irland profitierten von niedrigen Zinsen im Verhältnis zu ihrer Wachstumsrate; ihre Schuldenstandsquoten waren zwar stabil, hingen aber von der bestehenden Differenz zwischen Zinssatz und BIP-Wachstum ab. 2007 machten die Finanzmärkte noch keinen Unterschied zwischen den Staatsschulden der einzelnen Euroländer (Abschnitt 5.1).

Im Euroraum machten sich jedoch durch unhaltbare makroökonomische Strategien zunehmende Ungleichgewichte zwischen zwei Ländergruppen bemerkbar: Neomerkantilistische Strategien führten in den „tugendhaften“ Ländern des Nordens $\mathrm{zu}$ steigender Wettbewerbsfähigkeit und riesigen Leistungsbilanzüberschüssen ( $8 \%$ des BIP in Deutschland und den Nieder- landen, $5 \%$ in Finnland, 3,5 \% in Belgien und Österreich). Die südlichen Länder hingegen verloren an Wettbewerbsfähigkeit und häuften Leistungsbilanzdefizite an (5,3 \% in Irland, 8,5 \% in Portugal, 9,5 \% in Spanien und 12,5\% in Griechenland), verursacht durch ungleichgewichtige Wachstumsstrategien und getrieben durch negative Realzinsen, Immobilienblasen und private Verschuldung (Deroose et al. 2004; Mathieu/Sterdyniak 2007). Die globalisierten Finanzmärkte förderten dieses Auseinanderdriften noch durch erleichterte Kreditvergabe an Länder mit großen Leistungsbilanzdefiziten.

Die für alle Euroländer einheitliche Geldpolitik bei gleichzeitig völlig unterschiedlichen Wachstums- und Inflationsraten führte $\mathrm{zu}$ immer größeren Disparitäten, die im Rahmen der engen wirtschaftspolitischen Vorgaben des Maastrichtvertrages nicht ausgeglichen werden konnten. Die EU-Kommission hielt sich strikt an den Stabilitäts- und Wachstumspakt (SWP), der starre Regeln ohne Berücksichtigung der wirtschaftlichen Lage

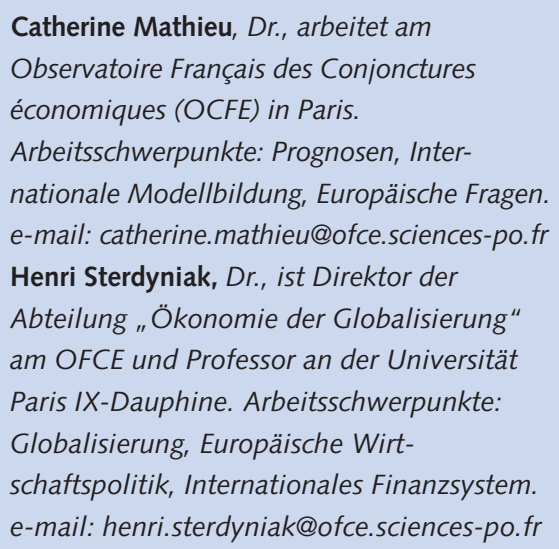

Übersetzung aus dem Englischen von Yvonne Silber und Achim Truger 
Tabelle 1: Indikatoren zur Stabilität der Staatsverschuldung 2007 - in \% des BIP -

\begin{tabular}{|c|c|c|c|c|c|c|}
\hline & $\begin{array}{c}\text { Staatlicher } \\
\text { Haushaltssaldo } \\
\text { (1) }\end{array}$ & $\begin{array}{l}\text { Staatlicher } \\
\text { Primärsaldo } \\
\text { (2) }\end{array}$ & $\begin{array}{l}\text { Nettoschulden- } \\
\text { stand } \\
(3)\end{array}$ & $\begin{array}{l}\text { Realzinssatz abzüglich } \\
\text { BIP-Wachstumstrend* } \\
\text { (4) }\end{array}$ & $\begin{array}{l}\text { Zur Stabilisierung der Schuldenstands- } \\
\text { quote erforderlicher Primärsaldo } \\
\qquad(5)=(3) *(4)\end{array}$ & $\begin{array}{l}\text { Schulden- } \\
\text { stabilitätslücke } \\
(6)=(2)-(5)\end{array}$ \\
\hline Deutschland & 0,2 & 2,6 & 42,2 & 2,0 & 0,8 & 1,8 \\
\hline Frankreich & $-2,7$ & $-0,2$ & 33,8 & 0,3 & 0,1 & $-0,3$ \\
\hline Italien & $-1,5$ & 3,2 & 87,0 & 0,9 & 0,8 & 2,4 \\
\hline Spanien & 1,9 & 3,0 & 18,5 & $-2,5$ & $-0,5$ & 3,4 \\
\hline Niederlande & 0,2 & 1,8 & 27,9 & 0,2 & 0,1 & 1,7 \\
\hline Belgien & $-0,2$ & 3,5 & 73,3 & 0,0 & 0,0 & 3,5 \\
\hline Österreich & $-0,5$ & 1,5 & 30,8 & 0,1 & 0,0 & 1,4 \\
\hline Griechenland & $-6,4$ & $-2,2$ & 79,8 & $-2,8$ & $-2,2$ & 0,0 \\
\hline Portugal & $-2,7$ & 0,2 & 42,7 & 0,5 & 0,2 & 0,0 \\
\hline Finnland & 5,2 & 4,6 & $-72,6$ & 0,1 & $-0,1$ & 4,7 \\
\hline Irland & 0,1 & 1,0 & $-0,3$ & $-4,0$ & 0,0 & 1,0 \\
\hline Euroraum & $-0,6$ & 2,0 & 43,3 & 0,3 & 0,1 & 1,9 \\
\hline $\begin{array}{l}\text { Vereinigtes } \\
\text { Königreich }\end{array}$ & $-2,7$ & $-0,8$ & 28,5 & $-0,3$ & $-0,1$ & $-0,7$ \\
\hline USA & $-2,8$ & $-0,9$ & 42,4 & $-0,6$ & $-0,3$ & $-0,6$ \\
\hline Japan & $-2,4$ & $-1,8$ & 81,5 & 0,9 & 0,7 & $-2,5$ \\
\hline
\end{tabular}

vorschreibt (Obergrenzen von $3 \%$ des BIP für die öffentliche Netto-Neuverschuldung und $60 \%$ des BIP für den öffentlichen Schuldenstand und als mittelfristiges Ziel einen ausgeglichenen Haushalt). Weder wurde die makroökonomische Politik gemäß der unterschiedlichen Lage der einzelnen Länder koordiniert, noch wurden makroökonomische Wachstumsstrategien entwickelt. Stattdessen sollten liberale Reformen durchgesetzt werden (Deregulierung von Güter-, Kapital- und Arbeitsmärkten, Kürzungen öffentlicher Ausgaben und Steuersenkungen).

In den Jahren 1999 bis 2007 wuchs die Wirtschaft im Euroraum mit 2,2 \% pro Jahr verglichen mit $2,9 \%$ in den USA und $2,8 \%$ im Vereinigten Königreich relativ langsam. Die Inflationsrate war mit 2,2 \% pro Jahr niedrig. Die Realzinsen entsprachen dem nominalen BIP-Wachstum, die Lohnquote sank um 2,3 Prozentpunkte, der Leistungsbilanzsaldo lag im Plus (0,5\% des BIP 2007), die Arbeitslosenquote blieb hoch (7,5 \% 2007). Für eine übermäßig expansive Finanzpolitik gibt es keinen Hinweis. Die Haushaltsdefizite dienten der Stützung der schwachen Wirtschaftsleistung und waren damit Ergebnis der schlechten makroökonomischen Politik.

\section{Finanzpolitik in der Krise}

Die aktuellen Haushaltsdefizite wurden durch die Wirtschaftskrise 2007 bis 2009 verursacht, die im Euroraum zu einem Einbruch von $8,5 \%$ der Wirtschaftsleistung gemessen am Vorkrisentrend führte. Die öffentlichen Finanzen stehen im Euroraum allerdings besser da als im Vereinigten Königreich, in den USA und in Japan. Für 2010 wird ein Anstieg der Haushaltsdefizite des Euroraums auf 6,3 \% des BIP erwartet, in Japan dagegen auf 6,5\%, im Vereinigten Königreich auf 10,5\% und auf $11,3 \%$ in den USA.

Ob es sich hierbei um strukturelle oder konjunkturelle Defizite handelt oder um den Effekt fiskalischer Impulse, ist schwer zu beurteilen. Das BIP fiel so abrupt, dass das potenzielle BIP schwer zu erfassen ist, ebenso wie der Ex-ante-Einfluss auf die Staatshaushalte wegen des enormen Wirtschaftseinbruchs (der nichtlineare Effekte auf die Steuereinnahmen hatte) und des dramatischen Verfalls von Vermögenswerten. Unter der Annahme, dass die Krise keinen Einfluss auf das potenzielle Wachstum hatte, waren die fiskalischen Impulse zwischen 2007 und 2009 (Tabelle 2) im Euroraum mit 1,7 \% des BIP weit geringer ausgeprägt als in den USA mit 3,9\% des BIP und im Vereinigten Königreich mit 3,6 \% des BIP.

\subsection{TECHNISCHE FRAGEN WERDEN ZU POLITISCHEN}

Die Bewertung der strukturellen öffentlichen Defizite und der erforderlichen Maßnahmen zur Wiederherstellung langfristig tragfähiger öffentlicher Finanzen hängen eng mit der Einschätzung der potenziel- len Wirtschaftsleistung zusammen. Die EU-Generaldirektion für Wirtschaft und Finanzen (GD ECFIN) hat ihre Schätzungen des potenziellen BIP und des Potenzialwachstums seit der Krise erheblich abgesenkt (Tabelle 3): Die Schätzung für das potenzielle BIP des Euroraums für 2009 wurde um 3,6 \% nach unten korrigiert; die Schätzung für das potenzielle Wachstum des Euroraums von 2009 bis 2011 von 2,2 \% vor der Krise auf nur noch $0,95 \%$. Zwar liegt 2010 das Euroraum-BIP $8,5 \%$ unterhalb des Vorkrisentrends, die Euroraum-Produktionslücke laut GD ECFIN liegt jedoch nur bei -2,9\%. Das würde bedeuten, dass die Finanzkrise das BIPNiveau dauerhaft um ca. 5,5\% gesenkt hat.

Die niedrigeren Potenzialschätzungen der Europäischen Kommission beruhen auf dem tatsächlichen BIP-Wachstum: In der Rezession wird weniger investiert, was zum Abnehmen der Produktionskapazitäten und des Arbeitskräftepotenzials führt (durch hohe Arbeitslosenquote entmutigte Arbeitnehmer geben die Jobsuche auf) und damit zu sinkender Produktivitätssteigerung. Aber was folgt daraus? Sollen die Mitgliedstaaten deswegen in Zukunft auf jede Ankurbelung der Nachfrage verzichten, oder sollten sie nicht besser durch hohes Wachstum die Produktionskapazitäten wieder erweitern und damit entmutigten Arbeitnehmern Arbeitsplätze anbieten und so ihre Produktivität bewahren?

Soll man das Wachstumsziel für den Euroraum in den kommenden Jahren bei 2,2 \% oder bei 1,0\% pro Jahr ansetzen? Immerhin besteht die Gefahr, dass ein 
Tabelle 2: Kumulierte fiskalische Impulse seit 2007 - in \% des BIP -

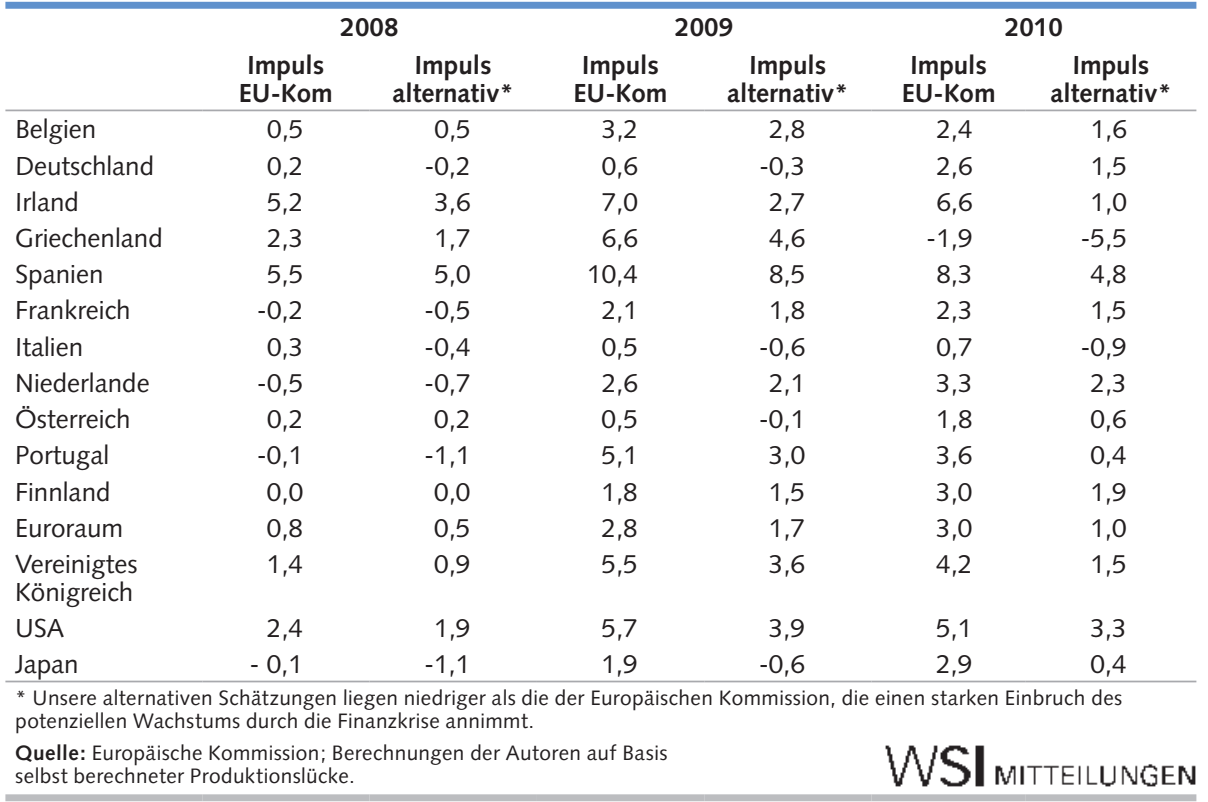

Tabelle 3: Schätzungen des potenziellen Wachstums und der Produktionslücke (EU-Kommission)

\begin{tabular}{|c|c|c|c|c|c|c|c|c|c|}
\hline \multirow[b]{3}{*}{ Schätzjahr } & \multirow{2}{*}{\multicolumn{2}{|c|}{$\begin{array}{c}\text { Produktionslücke } \\
\text { - in \% des BIP- } \\
2007\end{array}$}} & \multicolumn{7}{|c|}{ Potenzielles Wachstum - in \% - } \\
\hline & & & \multicolumn{2}{|c|}{$2000-07$} & \multicolumn{2}{|c|}{2008} & \multicolumn{2}{|c|}{2009} & 2010-11 \\
\hline & 2007 & 2010 & 2007 & 2010 & 2007 & 2010 & 2007 & 2010 & 2010 \\
\hline Deutschland & 0,3 & 1,7 & 1,4 & 1,3 & 1,8 & 1,1 & 1,9 & 0,9 & 1,4 \\
\hline Frankreich & $-0,3$ & 2,1 & 2,1 & 2,0 & 2,0 & 1,9 & 2,1 & 1,5 & 1,5 \\
\hline Euroraum & $-0,2$ & 2,2 & 2,1 & 1,9 & 2,1 & 1,4 & 2,2 & 0,9 & 1,0 \\
\hline $\begin{array}{l}\text { Vereinigtes } \\
\text { Königreich }\end{array}$ & $-0,1$ & 2,3 & 2,8 & 2,6 & 2,5 & 1,8 & 2,7 & 1,0 & 1,2 \\
\hline
\end{tabular}

Quelle: Europäische Kommission: Europäische Wirtschaft, Herbst 2007 und 2010.

WSI MITTELLUNGEN

niedriges Ziel zur selbst erfüllenden Prophezeiung wird, wenn bei Wachstumsraten über $1 \%$ eine restriktive Geldpolitik zum Einsatz kommt. Zudem sollen nach der Erklärung der EU-Kommission vom November 2009 die Mitgliedsländer 2011 ein „Haushaltskonsolidierungsprogramm“ starten, weil die BIP-Wachstumsprognosen mit $1,5 \%$ bedeutend höher liegen als das mit 1,0\% veranschlagte potenzielle Wachstum. Aus unserer Sicht sollte sich der Euroraum jedoch nicht mit einer Arbeitslosenquote von $10 \%$ abfinden und die Wirtschaftspolitik sich daher auf das Aufholen der krisenbedingten BIP-Verluste konzentrieren. Die Schätzungen der GD ECFIN taugen nicht dazu, Ziele für Wachstum und Haushaltsdefizite zu definieren.

\subsection{STRUKTURELLE DEFIZITE 2010}

Laut EU-Kommission lagen 2010 die konjunkturbereinigten Haushaltsdefizite im Euroraum bei $4,9 \%$ des BIP. Da die Euro- staaten mittelfristig ihre Haushalte ausgleichen sollen, ist dies gleichbedeutend mit Sparmaßnahmen für die Staatshaushalte in Höhe von 4,9 \% des BIP.

Unserer Meinung nach entstand der Anstieg der Defizite zum Teil durch kurzzeitige Konjunkturpakete und eine Überreaktion bei den Steuereinnahmen. Diese Faktoren sollten nicht in das strukturelle Defizit einfließen. Das Defizit des Euroraums läge 2010 bei $6,3 \%$ des BIP, von denen nach unseren Schätzungen 4,5 Prozentpunkte konjunkturell bedingt sind (inkl. 0,3 Prozentpunkte Überreaktion der Steuereinnahmen), 2,6 Prozentpunkte durch Zinszahlungen, 0,8 durch die Konjunkturpakete und 1,8 durch den strukturellen Primärüberschuss (Tabelle 4). Nehmen wir an, dass die öffentliche Verschuldung bei $80 \%$ des BIP stabilisiert werden soll. Der langfristige Realzinssatz im Euroraum lag zwischen 1997 und 2007 durchschnittlich 0,5 Prozentpunkte höher als das BIP-Wachstum. Der Euroraum müsste demnach einen strukturellen Überschuss von $0,4 \%$ des BIP erreichen. Im Jahr 2011 wurde ein strukturelles Defizit von $0,1 \%$ des BIP angesetzt; die Konsolidierungslücke betrüge mithin nur $0,5 \%$ des BIP. Zur Schließung einer solchen Lücke bedarf es keiner größeren finanzpolitischen Maßnahmen. Das konjunkturelle Defizit muss durch ein BIP-Wachstum ausgeglichen werden, das langfristig höher sein sollte als das potenzielle Wachstum von $2 \%$, um die negative Produktionslücke in Höhe von 8,5 \% des BIP zu reduzieren. Die Euroraumstaaten müssen sich auf das BIP-Wachstum konzentrieren und nicht auf die öffentlichen Haushalte.

\section{1}

\section{Finanzpolitische Exit- Strategien}

\subsection{SCHULDEN SENKEN?}

In der Krise wurden die Regierungen von IWF, OECD und der EU-Kommission gedrängt, massive Konjunkturprogramme aufzulegen. Heute rufen diese Institutionen nach restriktiver Finanzpolitik, obwohl die Produktionslücken wie erläutert immer noch sehr hoch sind und das Wachstum unterhalb des potenziellen Wachstums liegt. Es lässt sich schwer nachvollziehen, wie eine weltweite Sparpolitik keinen massiven negativen Einfluss auf das Wachstum haben soll.

Laut IWF (2010a; Cottarelli/Viñals 2009) sollen die entwickelten Ökonomien zu den öffentlichen Schuldenständen vor der Krise zurückkehren oder noch darunter bleiben, da ein positiver Zusammenhang gesehen wird zwischen dem Niveau der öffentlichen Schuldenstände und dem Realzinssatz, der seinerseits wiederum das Potenzialwachstum negativ beeinflusst. Eine Analyse der optimalen öffentlichen Verschuldung wird allerdings nicht geliefert. Die optimale Schuldenquote könnte heute möglicherweise höher als vor der Krise liegen, da die Privathaushalte zur Finanzierung ihrer Altersvorsorge langfristige, risikoarme Vermögenswerte vorziehen und sich die Aktienmärkte als zu riskant erwiesen haben. Der Zusammenhang zwischen öffentlicher Verschuldung und Zinssatz gilt zudem dann nicht, wenn die Zentralbanken für niedrige Zinsen sorgen und die Regierungen an den öffentlichen 
Defiziten festhalten, um die Wirtschaft zu stabilisieren, oder wenn die staatliche Schuldenquote deswegen hoch ist, weil die Unternehmen und ein Teil der Privathaushalte ihre Schulden abbauen und andere Privathaushalte mehr Finanztitel halten wollen.

In der Krise führten die Anstiege der öffentlichen Defizite und Schulden deswegen nicht zu höheren Zinsen, weil diese nur den privaten Schuldeneinbruch und den Anstieg der privaten Sparquote ausglichen. So fiel in den USA der Zinssatz für die 10-jährige Staatsanleihe von $4 \%$ im Juli 2008 auf 3,4 \% Mitte 2009 und lag im Dezember 2010 schließlich bei 2,9\%.

Der IWF verlangt von den entwickelten Ländern, zehn Jahre lang eine restriktive Finanzpolitik von $0,8 \%$ des BIP zu betreiben, ohne den Einfluss dieser Strategie auf das Wachstum zu untersuchen. Der IWF geht davon aus, dass die aktuellen öffentlichen Defizite überwiegend struktureller Natur sind. Damit aber wird der krisenbedingte Produktionsverlust zementiert. Der IWF geht offenbar implizit von einem kommenden privaten Nachfrageboom aus, den wir allerdings für unwahrscheinlich halten. Der IWF verschweigt, dass der angestrebte Ausgleich der Staatshaushalte kritisch davon abhängt, dass es zu einem solchen Boom kommt.

Die OECD verlangt darüber hinaus eine Drosselung der Staatshaushalte um $1 \%$ des BIP pro Jahr von 2012 bis 2017, um Ricardianisches Verhalten der Privathaushalte (Sparen in Erwartung von Steuererhöhungen) zu verhindern und die Finanzmärkte zu beruhigen - wohl wissend, dass diese Politik das Wachstum dämpfen wird. Es ist schwer zu verstehen, wie eine Politik, die eine lange Rezessionsperiode zur Folge hat, die Finanzmärkte beruhigen soll. Müssen wir trotzdem Ricardianisches Verhalten fürchten? Der aktuelle Anstieg der öffentlichen Schulden ist konjunkturbedingt und beruht nicht auf strukturellen öffentlichen Ausgaben oder Steuersenkungen. Steuererhöhungen sind also nicht angezeigt. Die Staatshaushalte sollten durch Produktionswachstum ausgeglichen werden. Paradoxerweise könnte ausgerechnet die Forderung von IWF und OECD nach einer raschen Konsolidierung zu Ricardianischem Verhalten der Bevölkerung führen.

Ironischerweise widerspricht gerade die IWF-Studie (2010b) dem Plädoyer für eine forcierte Haushaltskonsolidierung. Sie liefert eine sorgfältige Analyse

Tabelle 4: Öffentliche Finanzen im Euroraum - in \% des BIP -

\begin{tabular}{|c|c|c|c|c|c|}
\hline & 2007 & 2008 & 2009 & 2010 & 2011 \\
\hline BIP-Wachstum & 2,9 & 0,4 & $-4,1$ & 1,7 & 1,5 \\
\hline $\begin{array}{l}\text { Produktionslücke bezogen auf } \\
\text { Vorkrisentrend }\end{array}$ & 0,0 & $-1,8$ & $-8,1$ & $-8,5$ & $-9,0$ \\
\hline Staatlicher Finanzierungssaldo & $-0,6$ & $-2,0$ & $-6,3$ & $-6,3$ & $-4,6$ \\
\hline Nettozinszahlungen & 2,6 & 2,7 & 2,5 & 2,6 & 2,7 \\
\hline Konjunkturbedingter Saldo & & $-0,9$ & $-4,0$ & $-4,2$ & $-4,5$ \\
\hline Konjunkturprogramme & & $-0,2$ & $-1,3$ & $-0,8$ & \\
\hline Überreaktion der Steuereinnahmen & & & $-0,6$ & $-0,3$ & \\
\hline Struktureller Primärsaldo & 2,0 & 1,8 & 2,1 & 1,8 & 2,6 \\
\hline Struktureller Saldo & $-0,6$ & $-0,9$ & $-0,4$ & $-0,8$ & $-0,1$ \\
\hline Impuls durch Konjunkturprogramme (1) & & 0,2 & 1,1 & $-0,5$ & $-0,8$ \\
\hline $\begin{array}{l}\text { Impuls durch Veränderung des } \\
\text { strukturellen Primärsaldos (2) }\end{array}$ & & 0,2 & $-0,3$ & 0,3 & $-0,8$ \\
\hline Fiskalischer Impuls $(1+2)$ & & 0,4 & 0,8 & $-0,2$ & $-1,6$ \\
\hline $\begin{array}{l}\text { Kumulierte fiskalische Impulse gegenüber } \\
2007\end{array}$ & 0,0 & 0,4 & 1,2 & 1,0 & $-0,6$ \\
\hline
\end{tabular}

der finanzpolitischen Multiplikatoren. In der Vergangenheit führte eine Haushaltskonsolidierung in der Größenordnung von $1 \%$ des BIP zu einem Rückgang der Wirtschaftsleistung von $0,5 \%$ des BIP, allerdings bei gleichzeitigen Zinssenkungen, Wechselkursabwertungen und Außenhandelsgewinnen. Ausgehend von einer globalen Konsolidierung unter den entwickelten Ländern und Zinssätzen nahe Null läge der negative Effekt bei $2 \%$ des BIP. Wenn der finanzpolitische Multiplikator also weltweit bei 2,0 liegt, würde ein negativer Impuls von $0,8 \%$ des BIP das BIP um 1,6\% senken, die Haushaltssalden nicht verbessern (weil die Steuereinnahmen sinken) und die Schuldenstände ansteigen lassen.

OECD und IWF plädieren für strukturelle Reformen (mehr Wettbewerb auf den Gütermärkten, Abbau von Verzerrungen auf dem Arbeitsmarkt und bei den Steuern, jedoch keine Finanzmarktreformen), um die negativen Effekte restriktiver Finanzpolitik auszugleichen. Ähnlich empfiehlt Giavazzi (2009), einen potenziellen Rückgang der Wirtschaftsleistung durch Einkommensteuersenkungen und dadurch steigende Beschäftigungszahlen auszugleichen. Aber sind die niedrigen Beschäftigungszahlen in einer Situation von Massenarbeitslosigkeit tatsächlich auf den Arbeitsunwillen der Menschen zurückzuführen?

\subsection{SPARVORSCHLÄGE}

Haushaltskonsolidierungen sollen durch Kürzungen der öffentlichen und sozialen Ausgaben und nicht durch Steuererhöhungen erreicht werden, fordern internationale
Organisationen, ohne den sozialen Nutzen öffentlicher Ausgaben und deren Einfluss auf die Nachfrage zu berücksichtigen. Warum sollten die öffentlichen Ausgaben in Relation zum BIP wegen der Finanzkrise sinken? Sollten sich hinter fragwürdigen wirtschaftlichen Annahmen (die Kürzung von Sozialleistungen sei weniger schädlich für die Wirtschaftsleistung als Steuererhöhungen, weil dadurch trotz Besteuerung zur Arbeit angetrieben werde) ideologische Vorlieben verbergen? Die Finanzkrise hat die Risiken struktureller liberaler Reformen gezeigt: Sinkende Arbeitseinkommen und Sozialkürzungen zur Finanzierung von Steuersenkungen führten zu enormen Zuwächsen bei Finanztiteln und hohen Einkommen. Dadurch stieg die soziale Ungleichheit, die Nachfrage fiel, was dann durch untragbare Finanzblasen und steigende Verschuldung ausgeglichen werden musste.

Cottarelli/Viñals (2009) schlagen Kürzungen bei den gesetzlichen Gesundheitsund Rentenzahlungen vor. Zur Deckung der Differenz wären dann private Prämien an Finanzinstitutionen zu zahlen. Die Autoren liefern keinen Beweis, dass dies weniger kostspielig wäre. Es wäre eine Ironie des Schicksals, wenn ausgerechnet die Finanzkrise zur Förderung von Pensionsfonds führen würde, die sich in der Krise als riskant und unbeständig erwiesen haben.

Nach Annahmen der OECD erbringen Rentenreformen eine dreifache Dividende: eine Entlastung der öffentlichen Haushalte, ein Rückgang der privaten Sparquote (weil wegen der längeren Lebensarbeitszeit weniger für die Altersversorgung 
angespart werden muss) und ein daraus resultierender Nachfrage- und Beschäftigungsanstieg und steigendes Wachstum. Es könnte aber auch das Gegenteil eintreten: Wenn die Renten fallen, wird mehr gespart, auch aus Angst, lange vor Eintritt ins Rentenalter arbeitslos zu werden.

\subsection{DEFIZIT- UND SCHULDEN- VORSCHRIFTEN?}

OECD und IWF plädieren für „finanzpolitische Regeln und Expertenkomitees". Die Krise hat jedoch gezeigt, dass die Finanzpolitik keinen Automatismen unterliegen darf, sondern entschlossene und mutige Eingriffe durch die Regierungen erfordert, was von Expertenkomitees gerade nicht zu erwarten ist.

Merkwürdigerweise gehen die internationalen Institutionen davon aus, dass die Finanzpolitik durch willkürlich von den Regierungen festgelegte Defizit- und Schuldenvorschriften sich quasi von selbst regelt. Dabei sollte die Finanzpolitik sich eher darauf konzentrieren, das angestrebte Beschäftigungsniveau zu stabilisieren (oder zu erreichen) bei gleichzeitig zufriedenstellenden Inflationsraten und Zinssätzen. Staatsverschuldung und -defizite müssen auf dieses Ziel ausgerichtet werden. Als finanzpolitische Exit-Strategie sind daher niedrige Zinsen der Zentralbanken und Haushaltsdefizite, solange sie zur Stützung der Wirtschaft notwendig sind, angezeigt. Wenn die private Nachfrage in den kommenden Jahren kräftig steigt, wird das dann erforderliche Zurückfahren der öffentlichen Schulden großenteils automatisch vonstattengehen. Stagniert die private Nachfrage, werden weitere Haushaltsdefizite und ein höherer öffentlicher Schuldenstand akzeptiert werden müssen. Es hat keinen Sinn, Defizit und Schuldenstandsprojektionen unabhängig von der privaten Nachfragesituation anzustellen und sich über eine ausufernde Staatsverschuldung zu sorgen (Cecchetti et al. 2010; Becker et al. 2010): Die Staatsverschuldung wird hoch sein, solange es eine Nachfrage nach Staatsverschuldung gibt. Es gibt heute aber keine Anzeichen dafür, dass die öffentliche Verschuldung in Zukunft übermäßig hoch sein wird.

\subsection{LEHREN DER KRISE}

Die Debatte über finanzpolitische ExitStrategien sollte die Ursachen der Krise nicht ignorieren, namentlich die Risiken wachsender Ungleichheit und finanzieller Instabilität. Das Platzen von Finanzund Immobilienblasen, der Anstieg der privaten Verschuldung und das Scheitern liberaler und neo-merkantilistischer Strategien haben die öffentlichen Defizite in die Rolle des Wachstumsmotors gezwungen. Um diese Defizite zu senken, muss sowohl in neo-merkantilistischen als auch in angelsächsischen Ökonomien eine neue Wachstumsstrategie entwickelt werden, die auf Löhnen und Sozialleistungen basiert, auf einer neuen Industriepolitik mit dem Ziel einer ökologischen und nachhaltigen Wirtschaft. Ein Teil der Probleme der öffentlichen Finanzen vor der Krise ist dem Steuerwettbewerb durch die neoliberale Globalisierung zu verdanken. Die öffentlichen Defizite sollten durch Steuererhöhungen für hohe Einkommen, große Vermögen und Einkommen aus Kapitalund Immobilienrenditen sowie für den Finanzsektor ausgeglichen werden. Eine steuerpolitische Koordination und eine Bekämpfung der „Steuerparadiese“ sollten dies ermöglichen.

\section{Öffentliche Finanzen und
Finanzmärkte}

Seit Mitte 2008 haben die Finanzmärkte ein neues Beschäftigungsfeld gefunden: Staatshaushaltsdefizite und öffentliche Verschuldung. Alle entwickelten Länder, selbst die größten, stehen seither unter dem Verdacht, ihre Schulden nicht bedienen zu können (Tabelle 5). Banken, Ratingagenturen und Investmentfonds zeigen sich besorgt über die Nachhaltigkeit öffentlicher Finanzen und verlangen von den Ländern eine Reduktion ihrer Defizite durch Kürzungen öffentlicher Ausgaben.

\subsection{WIRTSCHAFTSPOLITIK UND STAATSSCHULDEN}

Die Regierungen stehen nun vor widersprüchlichen Anforderungen: Die Unterstützung der Wirtschaft einerseits und die Sicherstellung ihrer eigenen finanziellen Situation andererseits. Die Kapitaleigner bevorzugen im allgemeinen Finanztitel mit Substanz. Diese sind aber durch Finanzblasen entstanden. Nach dem Platzen von Blasen muss das Nachfragedefizit zwingend durch öffentliche Defizite und niedrige Zinsen ausgeglichen werden. Wenn die Finanzmärkte diese Logik nicht akzeptieren, wenn sie weiterhin steigende langfristige Zinsen als angebliche Risikoprämien für die Wirtschaftsförderung der Regierungen verlangen, wenn die Ansichten sich weiterverbreiten, dass „die Defizite von heute die Steuern von morgen sind“ und dass wir in einer defizitären Haushaltslage mehr sparen müssen, dann wird die Wirtschaftspolitik unwirksam und die Weltwirtschaft außer Kontrolle geraten.

In einer Weltwirtschaft mit riesigen Kapitalanhäufungen sind die Schulden automatisch ebenfalls riesig. Viele private und staatliche Akteure sind verschuldet und manche sind höher verschuldet als andere. Es gibt demnach immer Zweifel an der Schuldnersolvenz und Schuldenkrisen. Anleger wollen große Summen anlegen, sorgen sich dann aber um die Überschuldung der Kreditnehmer. Länder, Unternehmen und Privathaushalte als Kreditnehmer sind verletzbar, weil sie schwer verschuldet und von den Kapitalmärkten abhängig werden.

Märkte unterliegen dem Herdentrieb, ihre Erwartungen sind selbst erfüllend, und die Marktakteure wissen dies. Wenn sie ängstlich werden, fördert ihre Wachsamkeit das Risiko einer Krise. Der leiseste Zweifel an der Solvenz eines Schuldners kann zu Kapitalabflüssen und höheren Zinsen führen, die dann die Krise tatsächlich hervorrufen. Die aktuelle Staatsschuldenkrise ist nicht das Ergebnis übermäBiger öffentlicher Ausgaben, sondern der finanziellen Globalisierung.

Seit Mitte 2008 spekulieren die Finanzmärkte auf die Staatsschulden der EU-Länder. Sie haben realisiert, dass es im Rahmenwerk des Euroraums einen Webfehler gibt: Während die Regierungen anderer entwickelter Länder nicht zahlungsunfähig werden können, da sie sich stets über ihre Zentralbanken finanzieren können, haben die Euroländer sich dieser Möglichkeit entledigt. Der Europäischen Zentralbank ist es untersagt, einzelne Länder zu finanzieren. Artikel 125 der Römischen Verträge verbietet darüber hinaus die finanzielle Solidarität zwischen den Mitgliedsländern. Daraus folgt, dass die Finanzierung der Euroländer von den Finanzmärkten abhängt und nicht gesichert ist. Die Spekulation traf die verwundbarsten Ökonomien: Griechenland, Irland und Spanien. Diese Länder waren vor der Kri- 
se stark gewachsen, mussten jedoch ihre Wachstumsstrategien ändern. Damit wurde die Finanzkrise zur Eurokrise.

Die EU-Behörden waren in der Krise unfähig, wirksame Maßnahmen zu ergreifen. Ihre Reaktionen waren ängstlich, zögernd und widersprüchlich. Ihre Strategien bzw. das Fehlen von Strategien sind mit der Funktionsweise der Finanzmärkte nicht vereinbar. Mit dem Nähren von Zweifeln an der europäischen Solidarität und dem Einräumen der Möglichkeit des Staatsbankrotts einzelner Länder haben sie die Spekulation noch angeheizt. Die Euroländer und die EZB hätten unbegrenzte Kreditlinien für die bedrohten Länder einräumen und im Gegenzug die mittelfristige Konsolidierung dieser Staatshaushalte, kurzfristig jedoch keine zu harten Einschnitte verlangen sollen. Eine solche Entschlossenheit wäre stark genug gewesen, um die Spekulation einzudämmen. Das Thema „Moral hazard“ - moralisches Fehlverhalten - sollte für eine Weile vergessen werden. Es hätte Erklärungen geben müssen, dass die Probleme dieser Staatshaushalte eine innere Angelegenheit des Euroraums sind, die die Mitgliedsländer gemeinsam lösen werden. Das Fehlen von Solidarität und Vertrauen unter den Mitgliedsländern verhinderte dies jedoch.

\section{GRIECHENLAND}

Ende 2009 korrigierte die neue griechische Regierung die Schuldenzahlen der alten Regierung nach oben. Dies war der Beginn der Vertrauenskrise gegenüber den griechischen Schulden. Die EU-Behörden und die anderen Euroländer reagierten zögerlich. Sie wollten nicht den Eindruck erwecken, dass die Euroländer die uneingeschränkte Unterstützung ihrer Partnerländer erwarten können. Sie wollten Griechenland abstrafen, weil das Land nie die Kriterien des Stabilitäts- und Wachstumspakts eingehalten und die Höhe seines Schuldenstands verschwiegen hatte. Im Mai 2010 kündigte die EZB schließlich an, weiterhin bedingungslos im Rahmen von Rückkaufsvereinbarungen griechische Staatsanleihen aufzukaufen. Die EU und die Euroländer vereinbarten einen Kredit von $100 \mathrm{Mrd}$. an Griechenland, jedoch zu einem weit höheren Zinssatz (zunächst 5,2, dann 5,8 \%) als derjenige für starke Länder (3\%). Griechenland musste sich verpflichten, sein Defizit zwischen 2009 und 2010 um $6 \%$

Tabelle 5: Jährliche Zinssätze von 10-jährigen Staatsanleihen und Credit Default Swaps (CDS) - in \% -

\begin{tabular}{|c|c|c|c|c|c|}
\hline & \multicolumn{2}{|c|}{ Juni 2007} & \multicolumn{3}{|c|}{ September 2010} \\
\hline & 10-Jahres-Zins & CDS & 10-Jahres-Zins & CDS & $\begin{array}{c}\text { Standard \& } \\
\text { Poor's Rating }\end{array}$ \\
\hline Deutschland & 4,50 & 0,04 & 2,25 & 0,50 & AAA/stabil \\
\hline Frankreich & 4,55 & 0,07 & 2,60 & 0,90 & AAA/stabil \\
\hline Italien & 4,65 & 0,18 & 3,90 & 2,08 & A+/stabil \\
\hline Spanien & 4,55 & 0,07 & 4,20 & 2,37 & AA/negativ \\
\hline Niederlande & 4,50 & 0,02 & 2,45 & 0,55 & AAA/stabil \\
\hline Belgien & 4,55 & 0,03 & 3,10 & 1,41 & $\mathrm{AA}+/$ stabil \\
\hline Österreich & 4,50 & 0,06 & 2,75 & 0,95 & AAA/stabil \\
\hline Griechenland & 4,65 & 0,20 & 10,80 & 7,36 & $\mathrm{BB}+/$ negativ \\
\hline Portugal & 4,60 & 0,08 & 6,50 & 4,12 & $\mathrm{~A}^{-} /$negativ \\
\hline Finnland & 4,50 & n.a. & 2,49 & n.a. & AAA/stabil \\
\hline Irland & 4,45 & 0,13 & 6,75 & 4,54 & AA/negativ \\
\hline Dänemark & 4,45 & 0,13 & 2,35 & 0,45 & AAA/stabil \\
\hline Vereinigtes Königreich & 5,30 & n.a. & 3,05 & 0,74 & AAA/negativ \\
\hline Schweden & 4,30 & 0,34 & 2,35 & 0,44 & AAA/stabil \\
\hline USA & 5,00 & 0,13 & 2,70 & 0,60 & AAA/stabil \\
\hline Japan & 1,85 & 0,23 & 0,95 & 0,97 & AA/negativ \\
\hline
\end{tabular}

des BIP zu reduzieren und um kumulierte 11,5 Prozentpunkte von 2009 bis 2014, was schwer zu erfüllen sein wird. Der IWF wurde in diesen Plan eingebunden, um zu demonstrieren, dass diese Bedingungen genauso hart sind wie die des IWF gegenüber den Entwicklungsländern. Der Plan signalisiert jedoch das politische Scheitern des Euroraums. Die Mitgliedsländer mussten einräumen, dass gegenüber dem IWF und den Finanzmärkten jedes Land für sich allein steht. Im Juni 2010 schnellte die Differenz zwischen griechischen und deutschen Zehnjahres-Staatsanleihen auf fast acht Prozentpunkte herauf.

\section{IRLAND}

Im November 2010 brach die Irlandkrise aus. Irland war einst der liberale Klassenbeste mit den niedrigsten Staatsausgaben im Euroland, den niedrigsten Steuern (Unternehmensteuern und Sozialabgaben), einem Haushaltsüberschuss 2006 von 2,5\% des BIP, besonders starkem Wachstum (dank Steuerwettbewerb und niedrigen Zinsen im Verhältnis zur Wachstumsrate, was zu einer Immobilienblase führte). Dann durchlief die Wirtschaft die absolute Finanzkrise, besonders durch das Platzen der Immobilienblase. Das Bankensystem kollabierte. Die öffentlichen Finanzen Irlands wurden von der Krise hart getroffen mit einem BIP-Verlust von $22 \%$ zum Vorkrisentrend. Irland beschloss, sämtliche Schulden seiner Banken zu garantieren und sein Haushaltsdefizit 2010 von 13,2 auf
32,3 \% des BIP zu erhöhen, um seine Banken zu refinanzieren. Die irische Staatsverschuldung wird deshalb voraussichtlich von $25 \%$ des BIP 2007 auf 114 \% 2012 steigen. Die Ankündigung dieser Defizitsteigerung löste eine neue Spekulationswelle gegen Irland aus. EU und IWF liehen Irland 85 Mrd. $€$ zu 5,8\% Zinsen. Dafür muss Irland ein staatliches Sparprogramm von $10 \%$ des BIP bis 2014 einhalten. Irland weigert sich weiterhin, Unternehmenssteuern und Sozialabgaben zu erhöhen, weil es diese als die beiden irischen Pfunde im EU-Wettbewerb betrachtet.

\section{DIE EUROPÄISCHE FINANZ- STABILISIERUNGSFAZILITÄT}

Am 10.5.2010 wurde in größter Not die Europäische Finanzstabilisierungsfazilität (EFSF) ins Leben gerufen und mit $750 \mathrm{Mrd}$. $€$ ausgestattet. Es wurde vereinbart, dass der Fonds auf drei Jahre angelegt ist, dass seine Inanspruchnahme von der Kürzung staatlicher Ausgaben abhängt und dass die Zinsen Risikoprämien enthalten. Dieser Plan konnte die Märkte nicht beruhigen. Deutschland stellte harte Bedingungen für seine Ausweitung. Länder, die den Anforderungen nicht entsprechen, sollen ihr Stimmrecht in EU-Gremien verlieren. Dies würde den Ausschluss von Mitgliedsländern ermöglichen. Hilfe für Länder in Schwierigkeiten wäre von der Einführung restriktiver, von EU und IWF diktierter Programme abhängig, ein $\mathrm{Me}$ chanismus zur geordneten Insolvenz eines 
Mitgliedslands unter Einbeziehung des privaten Sektors soll eingeführt werden. Ab 2013 soll die Ausgabe von Staatsanleihen eine kollektive Ausübungsklausel enthalten. Die Anleger müssen das Risiko einer nur teilweisen Rückerstattung im Falle von wirtschaftlichen Schwierigkeiten des ausgebenden Landes akzeptieren.

Diese Mechanismen wurden von den Finanzmärkten erneut als fehlende europäische Solidarität interpretiert. Die Märkte wurden in ihrem Glauben bestärkt, dass die Staatsschulden der Euroländer nicht garantiert sind, dass es demzufolge legitim ist, Risikoprämien zu verlangen und dass es sich lohnen könnte, auf den Schuldenausfall einzelner Länder zu wetten. Staatsschulden werden nicht mehr als risikolos angesehen und damit teurer werden. Staaten werden der dauernden Bewertung durch die Finanzmärkte unterworfen. Diese schreiben den Südländern die größten Schwierigkeiten bei der Refinanzierung in den nächsten drei Jahren zu, sollten diese Mechanismen in Kraft treten. Dabei ist schon die aktuelle Schuldenrückzahlung vom Zugang zu den Finanzmärkten abhängig. Also wurde das Rating der Staatsanleihen der Südländer herabgesetzt. Ende November 2010 lagen die Zinsen für deutsche Staatsanleihen bei 2,7 \%, die der niederländischen und finnischen bei $3 \%$, für Österreich und Frankreich bei 3,2 \% und $4 \%$ für Belgien, $4,7 \%$ für Italien, Spanien $5,5 \%$, Portugal 7,1 \%, Irland 9,3 \% und bei $11,9 \%$ für Griechenland.

Die Finanzmärkte haben ein Szenario errichtet, in dem Austeritätsmaßnahmen zu schwachem Wachstum und sozialen Unruhen führen, was für einige Länder zum Austritt aus dem Euroraum führen kann. Wenn ein Land unter hohen Zinsen, schwachem Wachstum und hoher Arbeitslosigkeit leidet und seine Politik der EU und anderen Mitgliedsländern unterwerfen muss, ohne jegliche Aussicht auf Erholung, könnte ein Austritt zur Alternative werden. Darüber hinaus könnten die Finanzmärkte möglicherweise die Glaubwürdigkeit der Rettungspläne infrage stellen, das deutsche Bundesverfassungsgericht eine Verletzung der EU-Verträge und die Bevölkerungen der Südländer die Austeritätsmaßnahmen ablehnen, was wiederum Deutschland zur Beendigung des Plans verleiten könnte. Die Glaubwürdigkeit dieses Szenarios wird durch die Schwäche der Euroländer und der EU-Behörden bestätigt, die bislang weder ihre vollständige Solidarität noch den Willen zu einer einheitlichen makroökonomischen Strategie für den Euroraum demonstriert haben.

\section{KORREKTUREN?}

Einige Ökonomen und Politiker wie JeanClaude Juncker und Yves Leterme schlagen die Einführung einer europäischen Schuldenagentur (EDA) vor, die Schuldtitel für alle Euroländer ausgeben soll. Deutschland ist gegen den Vorschlag, weil es weder höhere Zinsen zahlen noch zur Rettung anderer Mitgliedsländer gezwungen werden will. Die EDA würde die nationalen Finanzpolitiken kontrollieren müssen, hätte die Macht, von schwachen Ländern Risikoprämien zu verlangen und könnte sogar eine Refinanzierung verweigern. Es wäre ein noch strafferer SWP mit den gleichen Problemen. Wie soll die Entscheidung über ein zu hohes Haushaltsdefizit ausfallen, wenn ein Mitgliedsland behauptet, das Defizit zur Stabilisierung seiner Wirtschaft zu brauchen oder zur Rettung seiner Banken?

Die Finanzmärkte sollten nicht auf den Bankrott souveräner Staaten wetten dürfen. Die Zentralbanken müssen dazu verpflichtet werden, Staatsschulden zu finanzieren, auch im Euroraum. Wenn ein Land unter andauernder privater Nachfrageschwäche leidet, sollte die Zentralbank die Zinsen senken und die Regierung ein Haushaltsdefizit hinnehmen. Wenn die Finanzpolitik glaubwürdig ist und wenn Geld- und Finanzpolitik koordiniert vorgehen, sind die langfristigen Zinsen niedrig. Dies fördert das Wachstum und begrenzt die öffentliche Verschuldung. In einem System flexibler Wechselkurse führt eine solche Politik zur Abwertung der Währung und damit zu einer Stabilisierung. Im Gegensatz dazu wird die Wirtschaftspolitik gelähmt, wenn die Märkte einen Staatsbankrott antizipieren und hohe Zinsen verlangt werden. Das Risiko besteht, dass die Euroländer in Zukunft ihre Schulden nicht mehr erhöhen können, aus Angst, dass die Märkte höhere Zinsen verlangen. Finanzpolitik wird damit wirkungslos. Die Finanzmärkte dürfen nicht die Wirtschaftspolitik bestimmen, die Brandstifter den Feuerwehrleuten keine Befehle erteilen. Das Risiko eines Staatsbankrotts sollte bei Null liegen und die Zentralbank verpflichtet werden, die öffentlichen Schulden als letzte Instanz zu finanzieren. Sonst kann der Euroraum in einer globalen Finanzwelt nicht überleben.

\subsection{DIE STRATEGIEN DER EUROLÄNDER}

2010 stehen alle Länder vor der Wahl zwischen der Konsolidierung ihrer Haushaltsdefizite, um zu hohe Staatsverschuldung zu vermeiden, und einer expansiven Finanzpolitik, da die Erholung weiterhin schwach ist (Tabelle 6). Deutschland (1,8\% des BIP), Österreich $(0,4 \%)$ und Finnland $(0,4 \%)$ konnten ihre positiven konjunkturellen Impulse beibehalten und damit die Wirtschaftsleistung der ganzen EU stützen. Im Gegensatz dazu waren Griechenland mit -10,1 \% des BIP, Irland mit $-5,7 \%$, Spanien mit $-3,7 \%$ und Portugal mit $-2,6 \%$ gezwungen, eine strenge restriktive Finanzpolitik einzuführen. Für 2011 wird dagegen eine Verschärfung in fast allen Industrieländern erwartet: Die Sparmaßnahmen sollen 2,0 \% des BIP im Euroraum betragen, 2,6\% im Vereinigten Königreich und $1,7 \%$ in den USA.

Unter dem Druck von IWF und EUKommission müssen bedrohte Länder drastische und oftmals unüberlegte Pläne zur Bekämpfung ihrer Staatsschulden implementieren. Dies bedeutet für Griechenland staatliche Eingriffe in Höhe von $16 \%$ des BIP, $9 \%$ für Irland, 8,5 \% für Spanien und $8 \%$ für Portugal. Die Südländer sehen damit kurzfristig einem Einbruch ihrer Wirtschaftsleistung und einer langen Rezession und hoher Arbeitslosigkeit entgegen. Nach Schätzungen der EU-Kommission wird 2012 die Arbeitslosigkeit in Portugal $11 \%$ erreichen, $13 \%$ in Irland, $15 \%$ in Griechenland und $19 \%$ in Spanien. Das BIP werde im Euroraum 2010-2011 durchschnittlich um $1,6 \%$ p.a. steigen, aber nur um $0,4 \%$ in Irland, 0,3 \% in Spanien, 0,2 \% in Portugal und $-3,6 \%$ in Griechenland. Die Defizitziele würden wahrscheinlich nicht erreicht und die Länder unter noch höheren Zinszahlungen und geringeren Steuereinnahmen leiden. Die Schuldenquote wird steigen und noch strengere restriktive Maßnahmen zur Folge haben. Gleichzeitig würden die Nordländer durch den Druck der Kommission, in die Grenzen des Stabilitäts- und Wachstumspakts zurückzukehren, und aus Angst, dass ihre Kreditwürdigkeit von den Ratingagenturen herabgestuft wird, ihre Schulden zurückfahren.

Diese Programme werden das soziale Modell Europas tief beeinflussen. Der Stellenabbau bei den öffentlichen Bediensteten wird die Qualität von Bildung, Gesundheit und Versorgung verschlechtern, die 
gesetzlichen Renten werden sinken, das Rentenalter wird steigen, Zuschüsse für Familien gekürzt. Spanien kürzt Gelder für Arbeitslose und Familien, Irland seinen Mindestlohn und Deutschland sein Existenzminimum, alle Länder üben Druck nach unten auf die Löhne aus, um ihre Wettbewerbsfähigkeit zu steigern. Sogar das Vereinigte Königreich, die Niederlande, Frankreich und Deutschland, die nicht direkt von der Spekulation betroffen sind, haben Kürzungen der staatlichen Ausgaben bei Sozialausgaben, Arbeitsförderung und Einkommen öffentlicher Bediensteter angekündigt. Solange die Wirtschaft in der EU nicht auf einem akzeptablen Weg zur Vollbeschäftigung ist, hätten diese Restriktionen nicht durchgeführt werden dürfen. Die Nordländer sollten stattdessen zum Ausgleich der restriktiven Politik in den Südländern ihre staatlichen Ausgaben erhöhen.

\section{Ein neues wirtschaftliches Rahmenwerk?}

Die Schuldenkrise stärkt politisch die Befürworter automatischer fiskalischer Regeln, da sie sich auf die Bedrohungen durch die Finanzmärkte berufen können und auf den Druck Deutschlands, das ein Erstarken des EFSF durch Stärkung des Stabilitätsund Wachstumspakts ausgleichen will. Der Fokus auf die Griechenlandkrise lenkt dabei von der Finanzkrise als eigentlichem Problem ab.

Die Höhe der Staatsverschuldung wird das Risiko einer Aufsicht der öffentlichen Finanzen durch die Finanzmärkte weiter erhöhen. Dies ist sehr unbefriedigend, da die Finanzmärkte keine makroökonomische Perspektive haben; sie sind prozyklisch (weil sie in schlechten Zeiten sparen wollen) und selbsterfüllend. Über die erforderliche Wirtschaftspolitik haben sie ihre eigenen Ansichten, aber wie weit treffen diese zu? Das Risiko besteht, dass die Euroländer große Anstrengungen unternehmen, der Macht der Finanzmärkte zu entkommen, indem sie ihre Haushaltsdefizite zu stark beschneiden, was die Wirtschaft für lange Zeit schwächen wird. Ihre Fähigkeit zu aktiver Finanzpolitik wird eingeschränkt. Was wäre geschehen, wenn die Regierungen sich 2009 geweigert hätten, den Banken zu helfen, damit sie

Tabelle 6: Fiskalische Impulse* 2010-2012 - in \% des BIP -

\begin{tabular}{|c|c|c|c|}
\hline & 2010 & 2011 & 2012 \\
\hline Deutschland & $2,0(1,8)$ & $-0,6(-0,7)$ & $-0,9(-0,8)$ \\
\hline Frankreich & $0,1(-0,3)$ & $-1,5(-1,9)$ & $-0,5(-0,9)$ \\
\hline Italien & $0,2(-0,5)$ & $-0,3(-1,1)$ & $-0,3(-0,5)$ \\
\hline Spanien & $-2,1(-3,7)$ & $-2,9(-4,4)$ & $-0,5(-2,0)$ \\
\hline Niederlande & $0,7(0,2)$ & $-1,9(-2,6)$ & $-0,9(-1,5)$ \\
\hline Belgien & $-0,9(-1,2)$ & $0,0(-0,4)$ & $0,4(-0,5)$ \\
\hline Österreich & $1,0(0,7)$ & $-0,5(-0,8)$ & $-0,2(-0,6)$ \\
\hline Portugal & $-1,6(-2,6)$ & $-3,7(-3,9)$ & $0,2(-0,9)$ \\
\hline Finnland & $1,1(0,4)$ & $-1,1(-1,6)$ & $-0,5(-1,0)$ \\
\hline Irland** & $-0,4(-1,7)$ & $-1,7(-4,7)$ & $-1,0(-3,0)$ \\
\hline Griechenland & $-8,5(-10,1)$ & $-3,5(-5,4)$ & $-0,5(-2,2)$ \\
\hline Euroraum & $0,7(-0,4)$ & $-1,8(-2,0)$ & $-0,5(-1,0)$ \\
\hline Vereinigtes Königreich & $-1,3(-2,1)$ & $-1,9(-2,6)$ & $-1,8(-2,5)$ \\
\hline USA & $-0,4(-0,6)$ & $-1,1(-1,7)$ & $-1,6(-2,1)$ \\
\hline Japan & $1,0(0,9)$ & $-0,4(-1,0)$ & $-0,1(-0,7)$ \\
\hline \multicolumn{4}{|c|}{$\begin{array}{l}\text { *Die erste Ziffer gibt die GD ECFIN-Schätzung wieder, die zweite unsere Schätzung, bezogen auf den Vorkrisentrend. } \\
{ }^{* *} \text { Ausgaben zur Bankenrettung nicht enthalten. }\end{array}$} \\
\hline \multicolumn{3}{|c|}{$\begin{array}{l}\text { Quelle: Wirtschaftsprognosen der Europäischen Kommission (Herbst 2010) } \\
\text { und OECD Economic Outlook (November 2010), Berechnungen der Autoren. }\end{array}$} & MITTEI \\
\hline
\end{tabular}

sich nicht auf den Finanzmärkten hätten refinanzieren müssen?

\subsection{NATIONALE PERSPEKTIVEN}

Die Krise erfordert ein Umdenken der EUWirtschaftspolitik und der Koordination der nationalen Staatsführungen. Denn die aktuelle Finanzspekulation profitiert vom Scheitern des europäischen wirtschaftspolitischen Rahmens.

Deutschland hat bereits eine neue „Schuldenbremse” im Grundgesetz eingeführt, die ein strukturelles Defizit von größer als 0,35 \% des BIP ab 2016 verbietet. Dabei wird auf Bundesebene das konjunkturelle Defizit mit der Methode der EUKommission ermittelt, was sehr fragwürdig ist, wie wir bereits gesehen haben. Nach dieser Methode hätte das deutsche strukturelle Defizit seit 1974 fast jedes Jahr oberhalb dieser Grenze gelegen. Ist es jedoch zu glauben, dass ein Land mit einem Leistungsbilanzüberschuss von derzeit 6,5\% des BIP, einer Arbeitslosenquote von ca. $8 \%$ und einer Inflationsrate von nur $1,5 \%$ an einem übermäßigen Haushaltsdefizit leidet? Sollte das Wachstum zukünftig geringer ausfallen, werden die Beschränkungen der Finanzpolitik vor allem von den Schätzungen des potenziellen Wachstums abhängen. Langfristig wird - unter der Annahme eines Wachstumstrends für Deutschland von $3 \%$ p.a. in nominalen Werten - ein Defizit von $0,35 \%$ des BIP die Staatsverschuldung auf $12 \%$ des BIP zurückfahren.

Aus deutscher Sicht soll der Stabilitäts- und Wachstumspakt gestärkt wer- den. Die Euroländer sollen zum schnellen Ausgleich ihrer öffentlichen Finanzen gezwungen werden und ebenfalls die deutsche Schuldenbremse einführen. Die nationalen Finanzpolitiken sollen von der EZB oder von unabhängigen Expertengremien überwacht werden. Länder, die diese Einschränkungen ablehnen und eine zu lasche Politik betreiben, sollen aus dem Euroraum ausgeschlossen werden. Die Länder sollen sich auf strukturelle Reformen und Wettbewerbsfähigkeit konzentrieren, um ihr Wachstum anzuregen.

Aus der Sicht Frankreichs soll eine koordinierte Wirtschaftspolitik zu einer makroökonomischen Strategie führen, die überwiegend auf Wachstum und Beschäftigung abzielt. Die strengen Regeln des Stabilitäts- und Wachstumspakts sollen durch einen koordinierten Prozess ersetzt werden, der die wirtschaftlichen Umstände berücksichtigt (Inflation, Arbeitslosigkeit und Leistungsbilanzen, Löhne und Finanzpolitik sowie nationale Unterschiede). Eine solche Strategie wird nicht leicht einzuführen sein. Die Euroländer werden sich weigern, ihre Macht ohne Sicherheiten an die EU abzutreten. Überdies haben sie unterschiedliche Ansichten über die richtige Wirtschaftssteuerung: Die Deutschen müssten überzeugt werden, ihre Löhne relativ zu erhöhen, Spanier und Griechen dagegen davon, ihre Löhne relativ zu senken. 


\subsection{DIE SICHTWEISE DER EU- KOMMISSION}

In der Krise ordnete die Kommission für 24 der 27 EU-Länder das Verfahren bei übermäßigem Defizit (EDP) an. Die Regeln des Stabilitäts- und Wachstumspakts wurden 2009 und 2010 zwar flexibel gehandhabt, die Krise hat jedoch offenbart, dass diese Regeln ungeeignet sind.

Am 12.5.2010 veröffentlichte die Kommission die Mitteilung: „Verstärkung der wirtschaftspolitischen Koordinierung“. Entgegen allen Belegen wird darin behauptet, dass „die Regeln und Prinzipien des Stabilitäts- und Wachstumspakts maßgebend und gültig" sind. Die Euroländer müssten nur gezwungen werden, diese auch anzuwenden.

Am 30. Juni schlug die Kommission ein „Europäisches Semester“ vor: Im ersten Halbjahr sollen alle Euroländer der Kommission und dem Europäischen Rat ihre kurz- und mittelfristigen Finanz- und ihre strukturellen Reformpläne vorlegen, vor der Abstimmung durch die nationalen Parlamente im zweiten Halbjahr. Diese werden dadurch mehr oder weniger den Entscheidungen auf EU-Ebene unterworfen. Für eine koordinierte Wirtschaftsstrategie wäre ein solcher Dialog sicherlich hilfreich, es besteht jedoch die Gefahr, dass dadurch mehr Druck in Richtung Sparmaßnahmen und liberale Reformen aufgebaut wird: Vielen Ländern wurde das EDP auferlegt, ihnen wurden jedoch keine Spielräume für eine expansive Finanzpolitik oder bei der Lohnentwicklung eingeräumt.

Am 29.9.2010 präsentierte die Kommission ein Paket von Vorschlägen mit dem Ziel einer wirksameren europäischen Koordinierung der Wirtschaftspolitik. Diese würden die Autonomie der Länder einschränken und ihre Befähigung zur Stabilisierung der Wirtschaft untergraben. Stattdessen sollen technokratische Regeln die Macht der Kommission stärken:

(1) Euroländer sollen sanktioniert werden, wenn die öffentlichen Ausgaben schneller wachsen als das vorsichtig geschätzte mittelfristige BIP-Wachstum (es sei denn, der Anstieg wird durch eine entsprechende Erhöhung der Einnahmen ausgeglichen oder der Haushaltssaldo liegt im Plus). Eine Unterstützung der Wirtschaftsleistung durch öffentliche Ausgaben wäre damit ausgeschlossen. Wer aber will ein „vorsichtig“ geschätztes Wachs- tum bemessen und muss der Euroraum überhaupt „vorsichtig“ sein?

(2) Länder mit einer Staatsverschuldung oberhalb von $60 \%$ des BIP unterlägen dem EDP, wenn die Differenz zwischen ihrer tatsächlichen Schuldenstandsquote und der $60 \%$-Grenze nicht um durchschnittlich $1 / 20$ p.a. in den letzten drei Jahren gefallen ist. Im Wirtschaftsabschwung ist es jedoch unmöglich, das Steigen dieser Quote zu verhindern. In einem Land mit einer Schuldenstandsquote von $90 \%$ des BIP und einer Inflationsrate von $2 \%$ bei einem Wachstum von $2 \%$ sollte das Defizit nicht höher als $2 \%$ des BIP liegen, bei einem Wachstum von $1 \%$ dürfte das Defizit dagegen nur bei $1 \%$ des BIP liegen.

(3) Länder mit zu schnell wachsenden öffentlichen Ausgaben und solche unter EDP sollen eine Einlage von 0,2 \% des BIP hinterlegen, die bei Nichterreichen der Vorschriften zur Geldbuße umgewandelt wird.

(4) Das Projekt behält die $3 \%$-Grenze für Defizite bei, das mittelfristige Ausgleichsziel sowie die Forderung für Länder mit einem strukturellen Defizit, dieses um mindestens $0,5 \%$ des BIP p.a. zu reduzieren.

(5) Die Länder sollen die EU-Regeln in ihre nationalen Regeln integrieren und nationale "unabhängige Haushaltsinstitutionen" einführen, um deren Einhaltung durchzusetzen.

(6) Die Kommission verlangt eine qualifizierte Mehrheit im Europäischen Rat zur Ablehnung der geplanten Maßnahmen und der Sanktionen.

(7) Die Kommission schlägt eine Überwachung von exzessiven makroökonomischen Ungleichgewichten anhand verschiedener Indikatoren vor (Wettbewerbsfähigkeit, Leistungsbilanzdefizit, öffentliche und private Verschuldung). Ein Verfahren bei einem übermäßigen Ungleichgewicht (EIP) soll eingeführt werden. Eine symmetrische Überwachung oder die Bestrafung von Ländern, die andere Euroländer durch zu strenge Finanz- und Lohnpolitik beeinträchtigen, sind damit allerdings nicht gesichert. Ebenso wenig ist eine koordinierte Ausgleichsstrategie für restriktive Politik in einigen Ländern und expansive Politik in anderen Ländern sichtbar, etwa ein Ausgleich der unterschiedlichen Wettbewerbsfähigkeit durch Lohnerhöhungen, Empfehlungen für Länder mit fallender Lohnquote, die Ausreichung umfangreicher EU-Kredite als Konvergenzhilfe für südliche und östliche Euroländer und zur Finanzierung von grünen Technologieunternehmen.

Die Vorschläge der Kommission sind ein weiterer Schritt in Richtung Entpolitisierung der Finanzpolitik. Sie werden die ständigen Spannungen zwischen der Kommission und den Euroländern zementieren. Das Projekt ist aus wirtschaftlicher Sicht sogar gefährlich: Alle Länder sollen für einen ausgeglichenen Haushalt sparen, was deren Wachstum schmälert und mit den Notwendigkeiten eines makroökonomischen Gleichgewichts nicht vereinbar ist. Die Euroländer sollen ihre Finanzpolitik an ein Expertengremium abtreten, obwohl die Krise die Notwendigkeit massiver und entschlossener finanzpolitischer Maßnahmen gezeigt hat.

Ende Oktober nahm der Europäische Rat die wirtschaftlichen Grundlagen dieses Projekts an, lehnte jedoch die Machtausweitung der Kommission ab. Sanktionen sollen automatisch erfolgen, jedoch nach Abstimmung des Rats mit qualifizierter Mehrheit: Ein sehr ambivalenter Kompromiss mit viel Zündstoff für künftige Spannungen.

\section{Vier Befürchtungen}

Die Krise zwang die Regierungen der EULänder und die Kommission zum Auflegen von Konjunkturpaketen, es wurden jedoch keine Lehren aus der Krise gezogen. Statt nach der Verantwortung der bisherigen Politik für den Ausbruch der Krise zu fragen, soll diese weitergeführt werden, als ob nichts geschehen wäre. Die Debatte über finanzpolitische Exit-Strategien ruft vier verschiedene Befürchtungen hervor:

(1) Die Probleme der öffentlichen Finanzen werden als Vorwand für harsche Ausgabenkürzungen benutzt, ein immer wiederkehrendes Ziel der europäischen Eliten. Für das Zusammenwachsen der EU wäre es eine Katastrophe, wenn diese mithilfe der EU-Behörden die Bedrohung durch 
die Finanzmärkte mit einer „SchockStrategie“ zur Durchsetzung restriktiver Wirtschaftspolitik, liberaler und antidemokratischer Reformen und für soziale Einschnitte benutzten. Die Aushöhlung des Sozialstaats ist sozial und wirtschaftlich gefährlich. Sie führt zum Absinken der Haushaltseinkommen und zum Ansteigen der Sparquote. Wie soll dann eine fallende Nachfrage ausgeglichen werden, etwa durch eine neue Finanzblase? Die privaten Haushalte würden dazu gezwungen, ihre Gesundheits- und Altersvorsorge individuell bei den für die Krise verantwortlichen Finanzinstitutionen einzukaufen. Soll das soziale Modell Europas untergraben werden, das sich in der Krise als so effektiv erwiesen hat?

(2) Es steht zu befürchten, dass die Sparmaßnahmen die Euroländer zwingen wer- den, wachstumsfördernde Ausgaben für Forschung und Entwicklung, Bildung, Förderung innovativer Industrien und eine ökologische Wirtschaft zu vernachlässigen.

(3) Der Anstieg von Defiziten und Schulden durch die Krise und die Bedrohung durch Ratingagenturen und Finanzmärkte wird die Regierungen dazu verleiten, zu starke und zu schnelle Restriktionen durchzusetzen, die die Erholung gefährden. Die Euroländer sollten u.E. stattdessen Defizite und Schuldenziele vergessen. Solange die Arbeitslosigkeit nicht auf annehmbarem Niveau in Richtung Vollbeschäftigung liegt, sollten keine Restriktionen zur Anwendung kommen. Europa kann sonst keine Wachstumsstrategien entwickeln und wird lange unter Stagnation und Massenarbeitslosigkeit leiden.
(4) Die Verschärfung des SWP, die Eingliederung finanzpolitischer Regeln in nationale Gesetzgebungen und fehlende Garantien für öffentliche Schulden im Euroraum werden eine aktive Finanzpolitik in Zukunft noch weiter erschweren.

Die Weltwirtschaft wird jedoch nicht durch Ungleichgewichte der öffentlichen Finanzen, sondern durch das Ansteigen von Spekulation auf den Finanzmärkten und das Fehlen einer globalen wirtschaftspolitischen Koordinierung gefährdet. Die Ausstiegsstrategien sollten daher die finanzielle Globalisierung infrage stellen und makroökonomische Strategien in neo-merkantilistischen wie in liberalen Ländern implementiert werden. Staatsanleihen sollten auf EU-Ebene garantiert, die makroökonomische Politik koordiniert und die Solidarität unter den Euroländern bestärkt werden.

\section{LITERATUR}

Becker S./Deuber, G./Stankiewicz, S. (2010): Staatsverschuldung in 2020, Deutsche Bank Research, März

Blanchard, O./Dell'Ariccia, G./Mauro, P. (2010): Rethinking Macroeconomic Policy, IMF Staff Position note 10 (03), 12. Februar, Washington, D.C.

Blanchard, O./Cottarelli, C. (2010): Ten Commandments for Fiscal Adjustment in Advanced Economies, IMF Webseite, 24. Juni Cecchetti, S./Mohanty, M./Zampolli, F. (2010): The future of public debt, prospects and implications, BIS Working Papers (300), Basel Cottarelli, C./Viñals, J. (2009): A Strategy for Renormalizing Fiscal and Monetary Policies in Advanced Economies, IMF Staff Position note, 22. September, Washington, D.C.

Delpla, J./Weizsäcker, J. von (2010): The blue bond proposal, Bruegel Policy Brief, Mai, Brüssel

Deroose, S./Langedijk, S./Roeger, W. (2004): Reviewing Adjustment Dynamics in EMU: From Overheating to Overcooling, Economics Papers (198), hrsg. von der Europäischen Kommission, Brüssel

Europäische Kommission/DG Ecofin (2009): Domestic Fiscal

Frameworks, Oktober, Brüssel

Europäische Kommission (2010a): Reinforcing economic policy

coordination, Mai, Brüssel

Europäische Kommission (2010b): Economic governance package, September, Brüssel

Giavazzi, F. (2009): Issues in the design of a fiscal exit strategy, Papier für den informellen Ecofin-Rat, Göteborg

Gros, D./Mayer, T. (2010): Towards a Euro(pean) Monetary Fund, CEPS Policy Brief (202), Brüssel
International Monetary Fund (IMF/IWF) (2010a): Navigating the Fiscal Challenges Ahead, Fiscal Monitor, Mai

International Monetary Fund (IMF/IWF) (2010b): Will it hurt?

Macroeconomic Effects of Fiscal Consolidation, in: World Economic Outlook, Oktober, S. 93-122

Mathieu, C./Sterdyniak, H. (2003): Reforming the Stability and Growth Pact, Document de travail de l'OFCE, Mai, Paris

Mathieu, C./Sterdyniak, H. (2006): A European Fiscal Framework designed for stability or growth?, in: Hein, E./Heise, A. Truger, A. (Hrsg.): European Economic Policies - Alternatives to Orthodox Analysis and Policy Concepts, Marburg

Mathieu, C./Sterdyniak, H. (2007): How to deal with economic divergences in EMU?, in: Intervention Journal of Economics 4 (2), S. 281-307

Mathieu, C./Sterdyniak, H. (2010): Global financial crisis: The French policy answer in an EU perspective, in: Dullien, S./Hein, E./Truger, A./ van Treeck, T. (Hrsg.): The World Economy in Crisis - The Return of Keynesianism?, Marburg, S. 155-190

Organisation für wirtschaftliche Zusammenarbeit und Entwicklung (OECD) (Hrsg.): Economic Outlook, diverse Ausgaben Organisation für wirtschaftliche Zusammenarbeit und Entwicklung (OECD) (2010): Preparing fiscal Consolidation, Paris

Tilford, S. (2010): How to save the Euro?, hrsg. vom Center for European Reform (CER), September

Von Hagen, J./Pisani-Ferry, J./Weizsäcker, J. von (2009): A European exit strategy, Bruegel Policy Brief, Oktober, Brüssel 